\title{
Knockdown of FOXO3 induces primordial oocyte activation in pigs
}

\author{
Mohammad Moniruzzaman, Jibak Lee, Mai Zengyo and Takashi Miyano \\ Graduate School of Agricultural Science, Kobe University, Nada-ku, Kobe 657-8501, Japan \\ Correspondence should be addressed to M Moniruzzaman; Email: monir@silver.kobe-u.ac.jp
}

\begin{abstract}
Mammalian ovaries are endowed with a large number of primordial follicles, each containing a nongrowing oocyte. Only a small population of primordial oocytes (oocytes in primordial follicles) is activated to enter the growth phase throughout a female's reproductive life. Little is known about the mechanism regulating the activation of primordial oocytes. Here, we found that the primordial oocytes from infant pigs (10- to 20-day-old) grew to full size at 2 months after xenografting to immunodeficient mice, whereas those from prepubertal pigs (6-month-old) survived without initiation of their growth even after 4 months; thereafter, they started to grow and reached full size after 6 months. These results suggest that the mechanism regulating the activation of primordial oocytes in prepubertal pigs is different from that in infant pigs. In this regard, the involvement of FOXO3, a forkhead transcription factor, was studied. In prepubertal pigs, FOXO3 was detected in almost all (94 $\pm 2 \%)$ primordial oocyte nuclei, and in infant pigs, $42 \pm 7 \%$ primordial oocytes were FOXO3 positive. At 4 months after xenografting, the percentage of FOXO3-positive primordial oocytes from prepubertal pigs had decreased to the infant level. Further, siRNA was designed to knock down porcine FOXO3. FOXO3knockdown primordial follicles from prepubertal pigs developed to the antral stage accompanied by oocyte growth at 2 months after xenografting. These results suggest that primordial oocytes are dormant in prepubertal pigs by a FOXO3-related mechanism to establish a nongrowing oocyte pool in the ovary, and that a transient knockdown of the FOXO3 activates the primordial oocytes to enter the growth phase.
\end{abstract}

Reproduction (2010) 139 337-348

\section{Introduction}

The mammalian ovary contains a large number of nongrowing small oocytes (primordial oocytes). Each oocyte is surrounded by a single layer of flattened granulosa cells. These granulosa cells, together with a small oocyte, are called the primordial follicle. Activation of primordial follicles causes the transformation of granulosa cells into a cuboidal shape (Lintern-Moore \& Moore 1979). The follicle at this stage is called a primary follicle. The granulosa cells then proliferate, and the follicles develop to the secondary, antral, and finally Graafian stages. The time point at which oocyte growth and follicular development begin depends on the species. In mice, oocyte growth begins within a few days after birth (Peters 1969, Hirshfield 1991), whereas in ruminants and primates, growth begins before birth (Russe 1983, Gougeon 2004). The ovaries of infant pigs contain a large number of primordial follicles and also a small population of developing follicles that contain growing oocytes. Primordial follicles represent more than $95 \%$ of the total follicular proportion in porcine ovaries at 2 weeks after birth (Black \& Erickson 1965, Oxender et al. 1979). The ovaries of prepubertal and adult animals possess numerous growing and fully grown oocytes in the developing and mature follicles respectively, as well as a number of nongrowing primordial oocytes as the future stock.

The mechanism regulating the initiation of oocyte growth is not well understood. Primordial follicles from newborn rodents are activated, and oocytes grow to full size in culture (Eppig \& O'Brien 1996). For humans and large domestic animals, however, in vitro growth of primordial oocytes is hardly applicable (Miyano 2003). The xenografting of ovarian tissues to immunodeficient animals can be a substitute for an effective long-term culture system. Mice homozygous for the Scid mutation lack both humoral and cell-mediated immunity due to the absence of mature T and B lymphocytes that render them able to accept the xenografts (Bosma et al. 1983, Bosma \& Carroll 1991). Gosden et al. (1994) have developed a method of xenografting mammalian follicles to severe combined immune deficiency (SCID) mice as a model for investigating the early stages of follicular development. Follicular development in xenografts in immunodeficient mice has been reported in different species, including cats (Gosden et al. 1994), 
sheep (Gosden et al. 1994), pigs (Kaneko et al. 2003), monkeys (Candy et al. 1995), marsupials (Mattiske et al. 2002), and humans (Oktay et al. 1998).

The duration of ovarian xenografting depends on the time course necessary for the follicular development of the donors. Primordial follicles developed to the antral stage at $45-75$ days after xenografting of the ovarian tissues from 20-day-old piglets (Kaneko et al. 2003). On the other hand, the development of primordial follicles from cows (Senbon et al. 2003) and prepubertal pigs (Moniruzzaman \& Miyano 2007) was not initiated at 6-8 weeks after xenografting. Primordial oocytes, therefore, are thought to differ between infant and prepubertal/adult animals. Accelerated development of xenografted ovarian follicles has been reported in younger pouch wallabies in comparison to older ones (Mattiske et al. 2002). We hypothesized that adult ovaries that contain growing oocytes require a mechanism to establish a pool of nongrowing oocytes as a reserve for the future, and that the growth of primordial oocytes in adult ovaries is restrained by a certain inhibitory mechanism. The recruitment of nongrowing oocytes might result from the release of the inhibitory mechanism that maintains the resting follicles in stasis (McGee \& Hsueh 2000).

It has been suggested that FOXO3, a forkhead transcription factor, regulates the activation of primordial follicles in the mouse ovary (Brenkman \& Burgering 2003). Forkhead transcription factors are characterized by the presence of a highly conserved, monomeric DNA-binding domain, also known as the forkhead box or FOX (Weigel \& Jackle 1990). In mammalian somatic cells, FOXO factors induce cell cycle arrest (in $\mathrm{G}_{1} / \mathrm{S}$ transition) and apoptosis (Brunet et al. 1999, Nakamura et al. 2000, Alvarez et al. 2001). FOXO3 expression has been observed in the nuclei of mouse and rat oocytes (Reddy et al. 2005), and Foxo3 ${ }^{-1-}$ mice show global follicular activation, which leads to the early depletion of ovarian follicles (Castrillon et al. 2003). These studies suggest that FOXO3 inhibits the primordial oocyte activation in newborn rodents. However, it has not been shown whether FOXO3 regulates the dormancy and reactivation of primordial oocytes in adult ovaries, since all oocytes are activated and depleted before puberty in the Foxo3-knockout mice.

The present study aimed to elucidate the primordial oocytes growth in pigs. We found that primordial oocytes in prepubertal pigs took much longer time to initiate growth than did those in infants. To determine the molecular mechanism underlying the difference between infant and prepubertal pigs, we examined the expression level of $\mathrm{FOXO} 3$ in primordial oocytes from infant and prepubertal pigs. Further, FOXO3 was knocked down by small RNA interference prior to xenografting to SCID mice in order to determine its effect on the activation of porcine primordial oocytes.

\section{Results}

\section{Growth of primordial oocytes from infant and prepubertal pigs}

Pig ovarian tissues dissected for xenografting contained primordial oocytes (Fig. 1A). Histological examination confirmed that the ovarian tissues dissected from both infant and prepubertal pigs contained mainly primordial follicles before xenografting (Figs 2A and B, 3A and C). The tissues did not contain any necrotic region. Each oocyte contained a large spherical nucleus. In infant pigs, some of the oocytes were gathered together, making a cluster- or nest-like appearance (Fig. 2A). In prepubertal pigs, the oocytes were scattered uniformly throughout the tissues (Fig. 2B), with a few exceptions of 1-2 cluster formations in a few tissues. The average numbers of oocytes per tissue were higher in infant $(154 \pm 6 ; n=65)$ than in prepubertal pigs $(78 \pm 8$; $n=116$ ). The oocyte diameters were $<35 \mu \mathrm{m}$ in both the infant and prepubertal pigs before xenografting (Fig. 4A and C).

After 2 months, ovarian xenografts from infant pigs grew approximately three- to five-fold in comparison to their original sizes, and some large antral follicles were visible. Histological examination revealed the development of follicles at various stages, including antral follicles, in all types of recipients, i.e. male $(n=7)$, female $(n=7)$, and ovariectomized $(n=6)$ SCID mice (Figs 2E and 3B). Average numbers of follicles obtained in the xenografts were $116 \pm 8,108 \pm 5$, and $117 \pm 5$ in male, female, and ovariectomized SCID mice respectively. The developing follicles were evenly distributed throughout the xenografts. The xenografts were free from necrosis and were well vascularized. Some follicles were hemorrhagic. The corpus luteum was not observed.
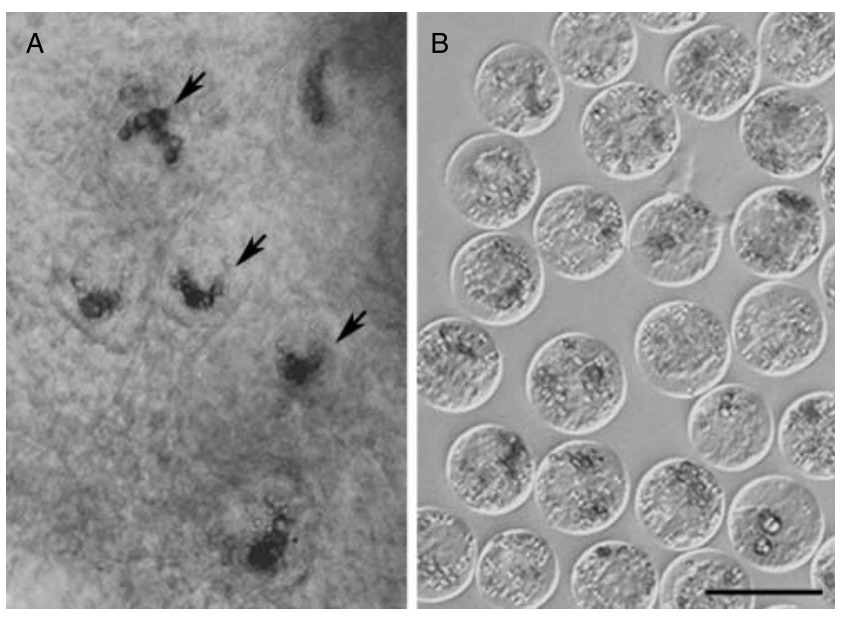

Figure 1 Primordial oocytes are visible in a porcine ovarian tissue under the inverted microscope. Arrows indicate the cytoplasmic lipid droplets at the peri-nuclear region of the oocytes (A). Primordial oocytes were collected enzymatically from ovarian tissues for western blot analysis (B). Scale bar represents $40 \mu \mathrm{m}$. 

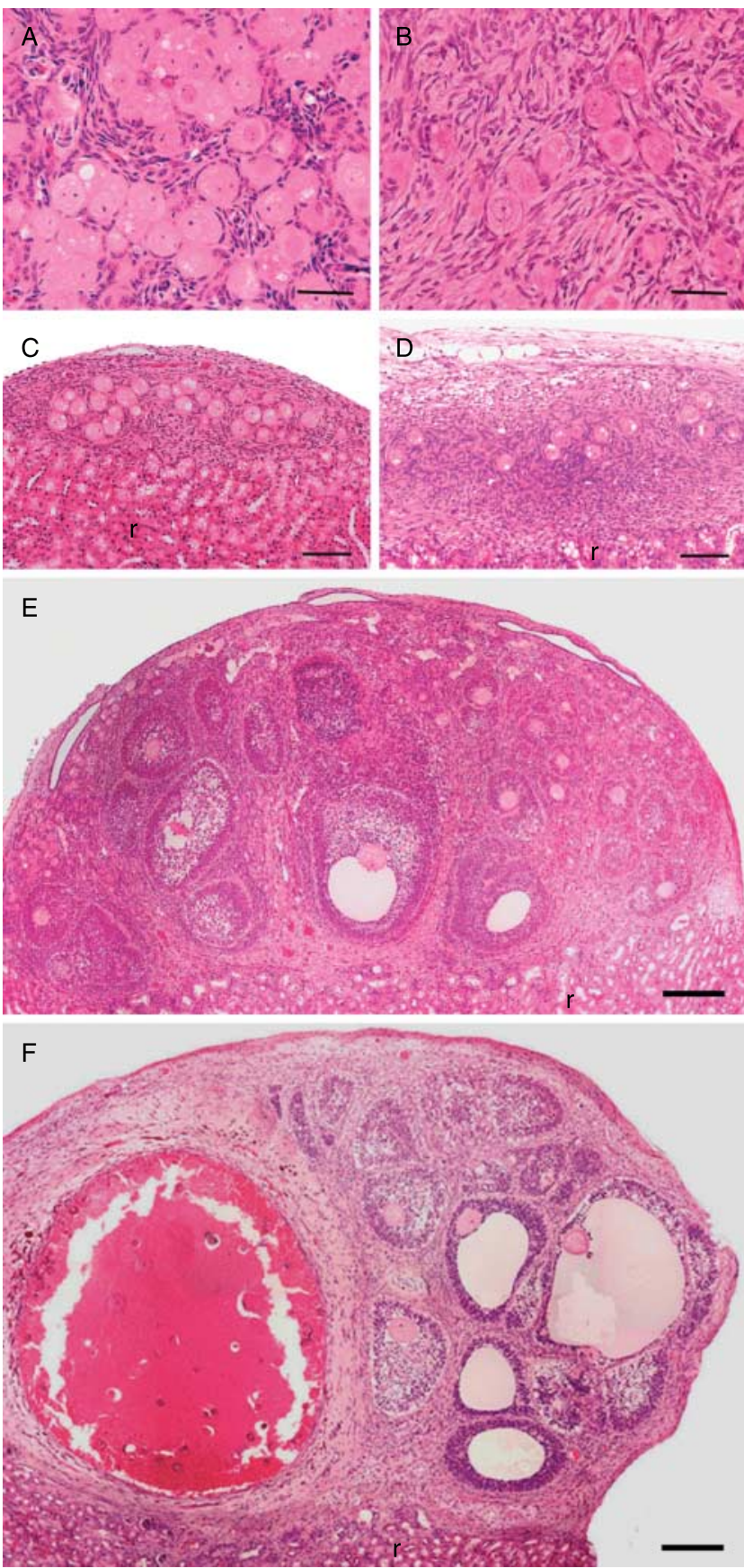

Figure 2 Histological sections of porcine ovarian tissues before and after xenografting. Ovarian tissues fixed in paraformaldehyde were embedded in methacrylate resin, serially sectioned by $5 \mu \mathrm{m}$, and stained with hematoxylin and eosin. Before xenografting, the ovarian tissues contained primordial follicles in both infant $(\mathrm{A})$ and prepubertal (B) pigs. At 2 months after xenografting, primordial follicles from infant pigs developed to the antral stage (E). In the same conditions, primordial follicles from prepubertal pigs had not started to develop at 2 (C) and 4 (D) months after xenografting. After 6 months, primordial follicles of prepubertal pigs developed to the antral stage (F).

' $r$ ' indicates mouse renal tissue $(C-F)$. Scale bars represent 40 (A and B), $100(\mathrm{C}$ and $\mathrm{D})$, and $200 \mu \mathrm{m}$ (E and F).
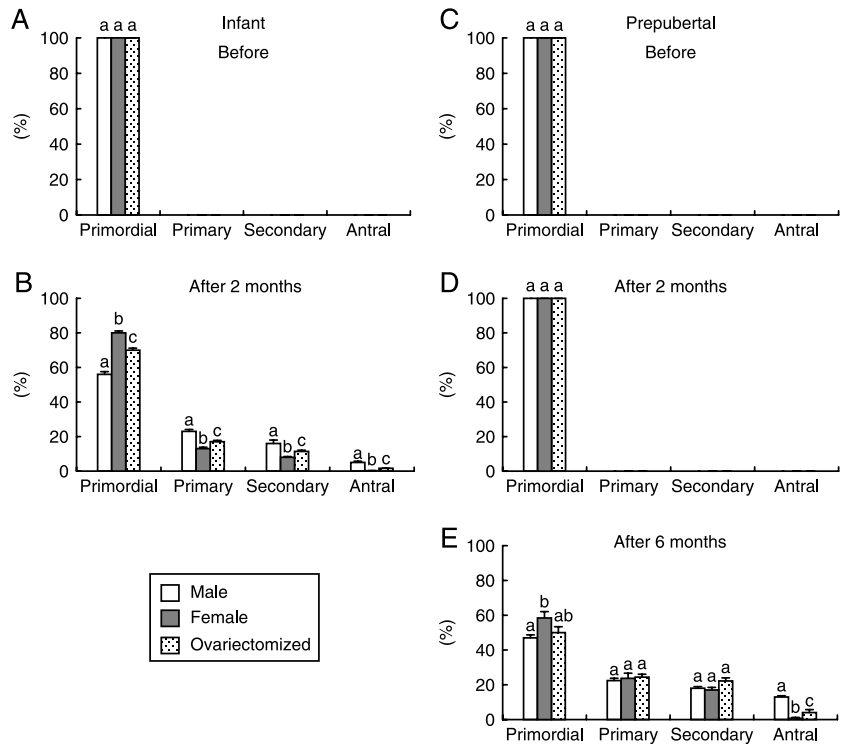

Figure 3 Distributions of different types of follicles in infant and prepubertal porcine ovarian tissues before and after xenografting. Ovarian tissues before and after xenografting were embedded in methacrylate resin, serially sectioned, and stained with hematoxylin and eosin. The number of different stages of follicles was recorded as described in Materials and Methods. Ovarian tissues from both infant and prepubertal pigs contained only primordial follicles before xenografting (A and C). After 2 months, the follicles of infant pigs developed to the various stages, including antral follicles (B), while the follicles of prepubertal pigs did not start to develop (D). After a prolonged period of 4 months, primordial follicles from prepubertal pigs remained in the similar stage. After 6 months, follicles developed to the antral stage (E). Each bar represents the mean \pm s.E.M. of follicles recorded from at least 11 separate tissues. Proportions of different types of follicles were compared among the recipients; male, female, and ovariectomized SCID mice. Bars with different letters within each category differ significantly $(P<0.05)$.

In the xenografts from prepubertal pigs, $54 \pm 2,66 \pm 3$, and $60 \pm 3$ primordial follicles survived in male, female, and ovariectomized mice respectively, but none of them developed to the primary stage or beyond after 2 months (Figs $2 \mathrm{C}$ and $3 \mathrm{D}$ ), irrespective of the type of recipient mice (male $n=6$, female $n=7$, and ovariectomized $n=6$ ). The diameters of the oocytes did not increase (Fig. 4D). The xenografts were well vascularized and free from necrosis. These results indicate that the initiation of oocyte growth and the pattern of follicular development are different between infant and prepubertal pigs. The duration of xenografting was prolonged in the case of prepubertals. At 4 months after xenografting, 52 \pm 3 , $47 \pm 2$, and $32 \pm 3$ oocytes survived in male $(n=3)$, female $(n=3)$, and ovariectomized $(n=3)$ mice respectively. In the xenografts, oocytes did not grow and follicles did not develop with a few exceptions. Three developing follicles (two primary and one secondary) were observed in three different xenografts, and the diameters of the oocytes ranged from 40 to $50 \mu \mathrm{m}$. All the other 15 xenografts contained only primordial follicles, and the diameters of the oocytes did not 

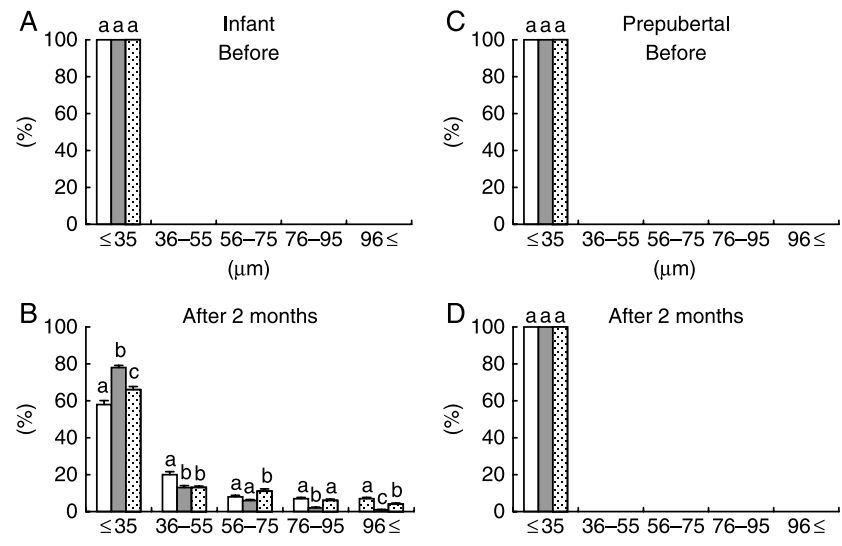

$(\mu \mathrm{m})$

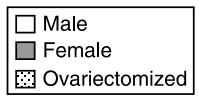

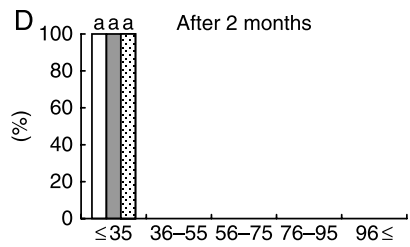

$(\mu \mathrm{m})$

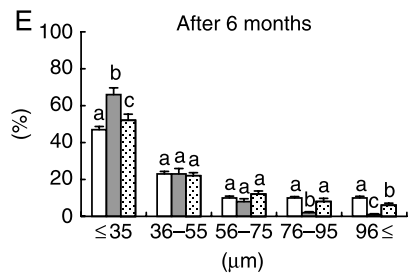

(a)

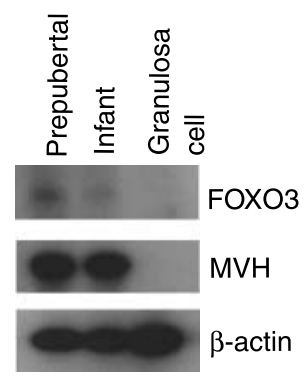

(b)

Infant
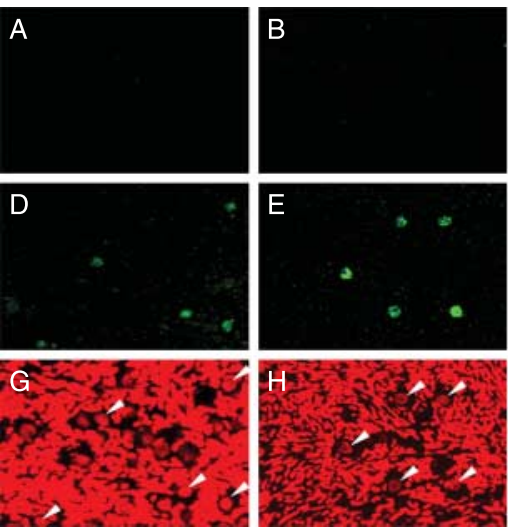

Figure 5 (a) Expression of $\mathrm{FOXO} 3$ in primordial oocytes from prepubertal and infant pigs by western blotting. Ovarian tissues were treated with actinase, and oocytes of around $30 \mu \mathrm{m}$ were collected. A number of 500 primordial oocytes or 5200 granulosa cells were prepared for each sample to be run on SDS-polyacrylamide gel. In each sample, a single FOXO3 band of $\sim 95 \mathrm{kDa}$ was detected by rabbit antiFOXO3 antibody (Cell Signaling Technology). FOXO3 level was higher in the primordial oocytes from prepubertal pigs than in those from infants. Mouse vasa homolog $(\mathrm{MVH})$ and $\beta$-actin were used as internal control. The experiment was repeated thrice with similar results.

(b) Immunolocalization of FOXO3 in primordial oocytes of infant and prepubertal pigs. Cryosections were treated with rabbit anti-FOXO3 antibody (Upstate Biotechnology) and Alexa Fluor 488-labeled antirabbit immunoglobulin antibody (green; D-F) and counterstained by $\mathrm{PI}$ (red; G-I). Serial sections were incubated without primary antibody as negative controls (A-C). The nuclei of some oocytes showed FOXO3 expression in infant porcine ovaries before xenografting (D). In contrast, all primordial oocytes were positively stained in prepubertal pigs (E). After 4 months of xenografting, some of the oocytes became FOXO3 negative (F). Arrow heads indicate the nuclei of oocytes showing FOXO3 signals. Scale bar represents $100 \mu \mathrm{m}$. 
Table 1 Reduction of FOXO3-positive primordial oocytes in prepubertal porcine ovarian tissues after 4 months of xenografting.

\begin{tabular}{lcccc}
\hline $\begin{array}{l}\text { Pig ovarian } \\
\text { tissues }^{\mathrm{a}}\end{array}$ & $\begin{array}{c}\text { Recipient } \\
\text { mice }\end{array}$ & $\begin{array}{c}\text { Number of } \\
\text { tissues } \\
\text { examined }\end{array}$ & $\begin{array}{c}\text { Number of } \\
\text { oocytes }\end{array}$ & $\begin{array}{c}\text { Number }(\%) \text { of } \\
\text { FOXO3- } \\
\text { positive } \\
\text { oocytes }\end{array}$ \\
\hline Infants & - & 4 & $79 \pm 7$ & $33 \pm 6\left(42 \pm 7^{*}\right)$ \\
$\begin{array}{l}\text { Prepubertals } \\
\text { Xenografts } \\
\text { from pre- } \\
\text { pubertals }\end{array}$ & $\begin{array}{c}\text { Male SCID } \\
\text { Female }\end{array}$ & 3 & $50 \pm 6$ & $47 \pm 4\left(94 \pm 2^{+}\right)$ \\
\hline
\end{tabular}

${ }^{*}{ }^{\dagger}$ Values with different superscripts are significantly different $(P<0.05)$. ${ }^{\mathrm{a}}$ Ovarian tissues measuring $\sim 1 \times 1 \times 0.5 \mathrm{~mm}$ were xenografted under the kidney capsule of SCID mice for 4 months. After fixation of ovarian tissues and xenografts, serial cryosections were reacted with antiFOXO3 antibody and Alexa Fluor 488-labeled secondary antibody. Sections were counterstained with $\mathrm{PI}$, and FOXO3-positive oocytes were counted throughout the tissues. Each value represents the mean \pm S.E.M.

in granulosa cells. FOXO3 was expressed in the nucleus of the primordial oocytes in both infant and prepubertal pigs (Fig. 5b). FOXO3 expression was reduced when sections were treated with the blocking peptide. This indicated that the antibody detected porcine FOXO3. Among a total of 317 primordial oocytes in infant pigs, FOXO3 expression was observed in $42 \pm 7 \%$ (Table 1 ). On the other hand, the signal was observed in almost all primordial oocytes (94 $\pm 2 \%$ in 201 observed oocytes) in prepubertal ovaries.

To examine whether $\mathrm{FOXO} 3$ expression is negatively correlated with the initiation of oocyte growth, immunostaining was performed in ovarian tissues from prepubertal pigs at 4 months after xenografting. It was thought that primordial oocytes started to grow at 4 months or later after xenografting. The percentage of FOXO3-positive oocytes decreased significantly in the xenografts (Fig. 5b-F and Table 1). Among 106 and 87 oocytes examined, the percentages of positively stained oocytes were $46 \pm 3$ and $63 \pm 6$ in male and female mice respectively.

\section{FOXO3 knockdown and growth of porcine primordial oocytes}

A partial nucleic acid sequence of porcine $\mathrm{FOXO} 3$ was determined to design effective siRNAs. PCR amplification of porcine mRNA generated a 1128-bp cDNA, which was highly similar to the corresponding region of mouse and human $\mathrm{FOXO} 3$ (Fig. 6). To determine the efficiency of siRNAs to knock down FOXO3, porcine somatic cells LLC-PK1 were transfected with siRNAs. The results of western blot analysis showed that the expression of FOXO3 was decreased in the cells treated with two siRNAs (No. 1 and No. 3 in Fig. 7a).

In preliminary experiments, different concentrations of transfectant reagent Lipofectamine 2000 and siRNAs were applied to knock down FOXO3 in primordial oocytes in ovarian tissues. Lower concentrations $(0.25-0.5 \%)$ of Lipofectamine 2000 did not result in significant change in $\mathrm{FOXO} 3$ expression in primordial oocytes in immunohistochemistry, while higher concentrations $(\geq 1.5 \%)$ caused the degeneration of oocytes. Finally, a $1 \%$ concentration was used for the transfection of siRNAs. To determine the efficiency of siRNAs to transfect the oocytes in ovarian tissues, Alexa Fluor 546labeled siRNAs were used. Oocytes and other ovarian cells in the tissues exhibited a red color, which indicated the penetration of siRNA into the oocytes (Fig. 7c). Western blot analysis of total protein extracted from ovarian tissues showed that the expression of FOXO3 decreased after treatment with $0.4 \mu \mathrm{M}$ siRNA No. 1 or siRNA No. 3, while control siRNA-treated tissues still expressed FOXO3 (Fig. 7b). Further, immunofluorescent staining confirmed the reduction of $\mathrm{FOXO} 3$ expression in oocytes in the ovarian tissues by siRNAs (Fig. 8a and Table 2). The percentage of FOXO3-positive oocytes in siRNA-treated tissues depended on the concentration of siRNAs. A lower concentration $(0.2 \mu \mathrm{M})$ of siRNAs did not interfere with $\mathrm{FOXO} 3$ expression in oocytes. FOXO3 expression was reduced in half of the primordial oocytes treated with $0.4 \mu \mathrm{M}$ siRNA, while almost all primordial oocytes were FOXO3 positive in the controls. Higher concentrations $(\geq 0.6 \mu \mathrm{M})$ of siRNAs caused the degeneration of oocytes (data not shown).

Following siRNA treatment, ovarian tissues containing primordial follicles were xenografted to SCID mice. In the histological examination, all tissues contained only primordial follicles before xenografting (Fig. 8G-I and Table 3). At 2 months after xenografting, oocyte diameters did not increase and follicles remained in the primordial stage in the controls (Fig. 8J and Table 3). Follicles developed to the secondary and antral stages in siRNA No. 1- and siRNA No. 3-treated tissues, accompaniment with the oocyte growth (Fig. 8K and $\mathrm{L}$ and Table 3).

\section{Discussion}

In mammals, a small population of primordial oocytes enters the growth phase at one time, while large numbers of them remain quiescent throughout a female's reproductive life (Hirshfield 1991). The initiation of primordial follicle development is known to be independent of gonadotropin stimulation (Fortune et al. 2000). Factors concerning the recruitment of primordial oocytes to enter the growth phase are not understood. Recently, xenografting of ovarian tissues or follicles has begun to be used as a method to study oocyte growth and follicular development. Kaneko et al. (2003) reported the development of primordial follicles to the antral stage at 45-75 days after xenografting ovarian tissues from 20-day-old pigs. The duration of follicular development in infant pigs in 


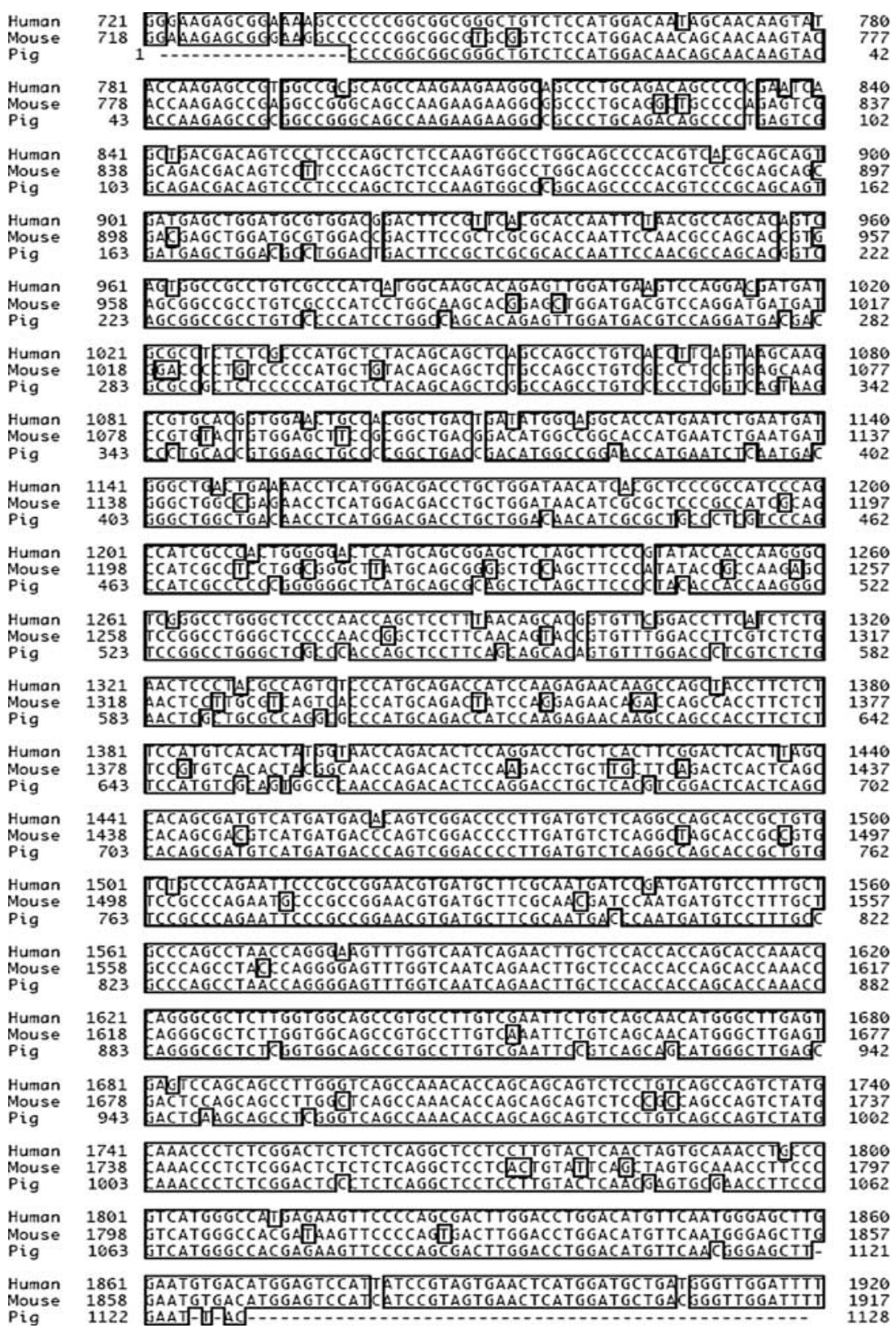

Figure 6 Partial sequence of porcine $\mathrm{FOXO3}$ cDNA, and its comparison with those of human and mouse. The common nucleotides among species are boxed. The sequence has been deposited to DDBJ (DNA Data Bank of Japan; accession number: AB281134). the present experiment is consistent with the result of Kaneko et al. (2003). However, in similar conditions, primordial follicles from prepubertal pigs had not started to develop even after 4 months. Similarly, our previous reports revealed that the primordial follicles from cows (Senbon et al. 2003) and prepubertal pigs (Moniruzzaman \& Miyano 2007) survived without initiating development at 6-8 weeks after xenografting. In the present study, a period of 6 months was required for development to the antral stage in the xenografts from prepubertal pigs. These results suggest that primordial oocytes in prepubertal pigs remain dormant for a long period before the growth is initiated.
Dormancy of primordial follicles is perhaps required to reserve nongrowing oocytes for their long reproductive life, because depletion of this oocyte reserve causes premature ovarian failure. The development of primordial follicles is prevented by some inhibitory factors in the ovary (McGee \& Hsueh 2000, Brenkman \& Burgering 2003). Castrillon et al. (2003) revealed that FOXO3 is a critical regulator that suppresses the initiation of primordial follicle development in the newborn mouse. In the present study, we observed FOXO3 in the nucleus of porcine primordial oocytes in agreement with previous results observed in mouse oocytes (Reddy et al. 2005). In prepubertal pigs, FOXO3 
was expressed in almost all primordial oocytes, and in infant pigs, a number of primordial oocytes did not exhibit FOXO3. The results of western blots reflected the higher proportion of FOXO3-positive primordial oocytes from prepubertal pigs than from infants. Further, at 4 months after xenografting, some oocytes from prepubertal pigs became FOXO3 negative, and some oocytes grew in the subsequent 2 months. These results imply that FOXO3 may be involved in the dormancy of primordial oocytes in prepubertal and perhaps adult porcine ovaries, and that the oocytes start to grow when they are released from the inhibitory action of FOXO3.

To clarify the involvement of FOXO3 in the suppression of oocyte growth in prepubertal pigs, we conducted a FOXO3 knockdown experiment using siRNAs. RNA interference by siRNAs is a well-established method for gene knockdown in cultured cells (Gampel \& Mellor 2002, Abdelrahim et al. 2003), and has recently been (a)

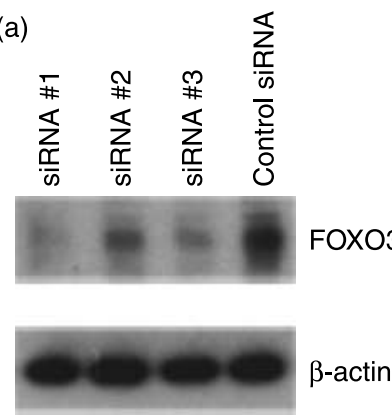

(c)
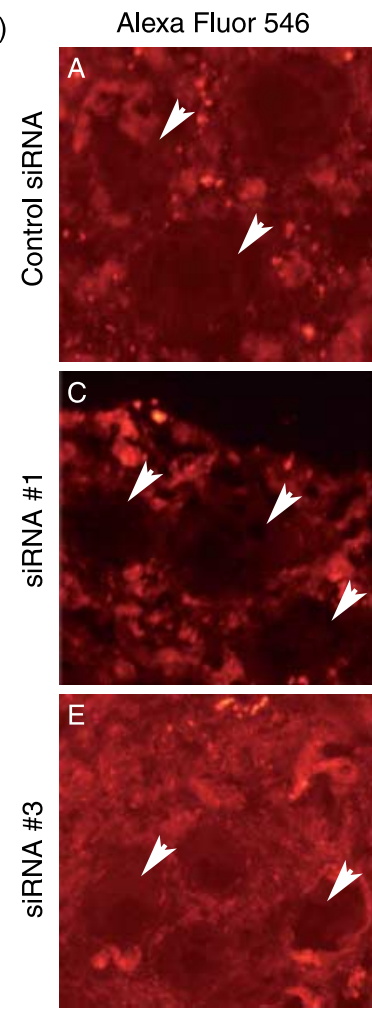

(b)
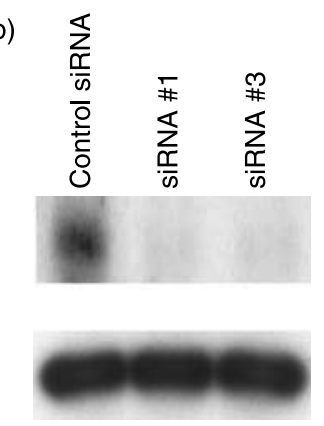

DAPI

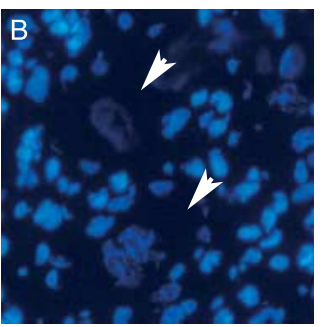

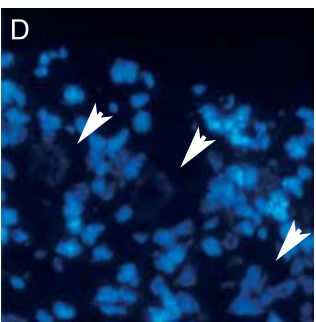

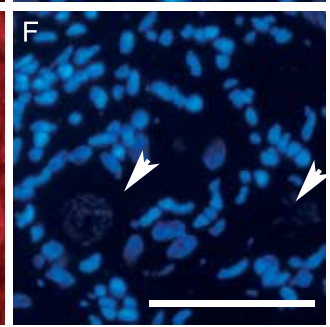

applied for oocytes (Lee et al. 2008), cultured hamster ovaries (Wang \& Roy 2006), and fetal mouse ovaries (Yamaguchi et al. 2006). In the present experiment, we knocked down FOXO3 in primordial oocytes in ovarian tissues from prepubertal pigs by siRNA transfection. Since siRNA mediates sequence-specific mRNA degradation, the transfected FOXO3 siRNAs worked transiently to knock down the target FOXO3 in the present experiment. After the knockdown of FOXO3, a proportion of primordial oocytes became activated and entered the growth phase in the xenografts. This result suggests that FOXO3 induces at least in part the dormancy of primordial oocytes in the prepubertal pig ovary. The ovaries of $\mathrm{FoxO}^{-1-}$ mice exhibit several fold increases in the number of developing follicles (Castrillon et al. 2003). At 8 days post partum, an enlargement of volume occurred in the majority of the oocytes, including those in primordial follicles. The pool of nongrowing oocytes was depleted in the ovaries of the mutant mice at 14 days post partum. Unlike the phenotypes of the Foxo3 ${ }^{-1-}$ mice, a proportion of follicles developed, while the majority of them remained in the primordial stage with nongrowing oocytes in the xenografts of FOXO3 siRNA-treated tissues in the present experiment. Here, the efficiency of siRNA to knock down the FOXO3 expression was low. FOXO3 knockdown might be related to the efficiency of siRNA transfection into the oocytes in ovarian tissues. However, Alexa Fluor 546-labeled FOXO3 siRNA indicated the penetration of siRNA into the oocytes as well as into other cells in the ovarian tissues. siRNA treatment caused the degeneration of oocytes in the ovarian tissues, although there was no significant difference between control siRNA and

Figure 7 (a) Porcine LLC-PK1 cells were treated for $6 \mathrm{~h}$ with three different FOXO3 siRNAs; No. 1: 5'-UGAGAUUCAUGGUUCCGGCCAUGUC-3', No. 2: 5'-UUCAGAGACGAGGGUCCAAACACUG-3', No. 3: 5'-UUCAAGCUCCCGUUGAACAUGUCCA-3', and control: 5'-GACCCGG AAGGUAC CUAAGCUAUCA-3'. Cells were further cultured in TCM-199 containing 5\% FCS for $36 \mathrm{~h}$ and were subjected to western blot analysis by using rabbit anti-FOXO3 antibody (Upstate Biotechnology). Expression of FOXO3 was decreased by two siRNAs (No. 1 and No. 3). Levels of $\beta$-actin were used as internal control. The experiment was repeated thrice with similar results. (b) Ovarian tissues containing primordial follicles from prepubertal pigs were treated with FOXO3 siRNAs (No. 1 and No. 3) for $6 \mathrm{~h}$ and further cultured in TCM199 containing 5\% FCS for $36 \mathrm{~h}$. Same amount of proteins extracted from treated tissues were separated in SDS-polyacrylamide gel, and western blotting was performed by anti-FOXO3 antibody (Cell Signaling Technology). FOXO3 level was decreased by FOXO3 siRNAs. Levels of $\beta$-actin were used as internal control. The experiment was repeated thrice with similar results. (c) Ovarian tissues containing primordial follicles from prepubertal pigs were treated with Alexa Flour 546-labeled FOXO3 siRNAs for $6 \mathrm{~h}$, cryosectioned, and observed under the fluorescence microscope. Red-colored cells are considered to be transfected with siRNAs (A, C, and E). Sections were counterstained with DAPI (blue; B, D, and F). The experiment was repeated thrice with similar results. Oocytes were indicated by arrow heads. Scale bar represents $60 \mu \mathrm{m}$. 
(a)
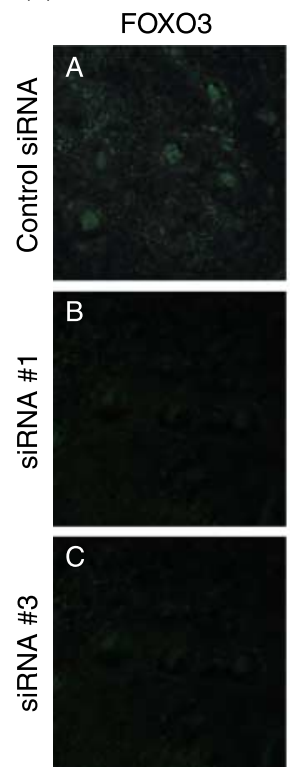
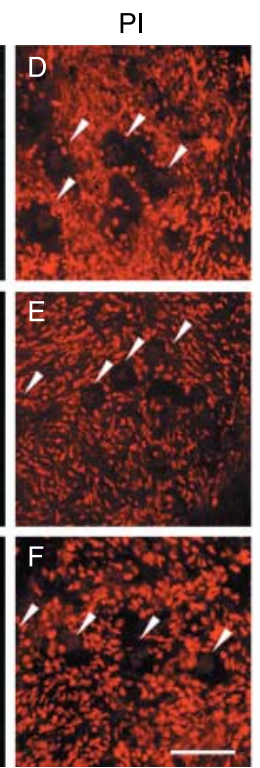

(b)

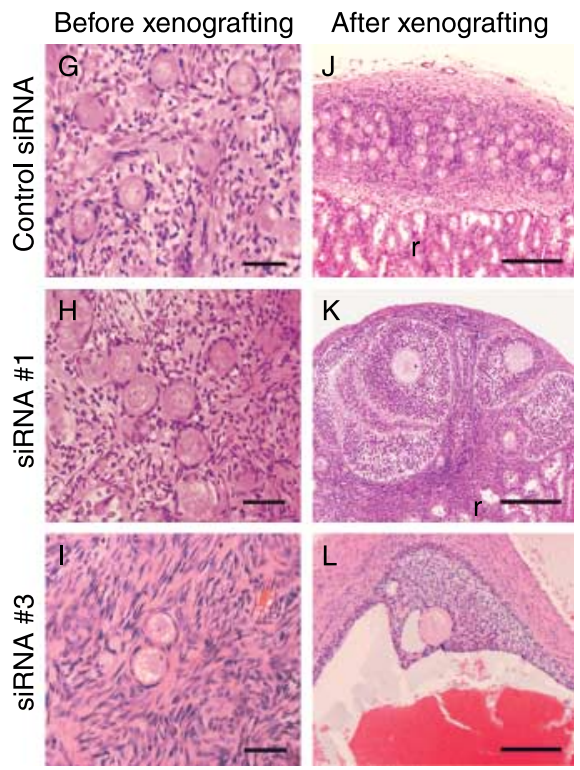

Figure 8 (a) Immunohistological examination of FOXO3 expression in siRNA-treated primordial oocytes in prepubertal pigs. Porcine ovarian tissues containing primordial follicles were treated with FOXO3 siRNAs (No. 1 and No. 3) for $6 \mathrm{~h}$, and further cultured in TCM-199 containing 5\% FCS for 36 h. Cryosections were treated with anti-FOXO3 antibody (Sigma) and Alexa Fluor 488-labeled secondary antibody (green; A-C) and counterstained with PI (red; D-F). The experiment was repeated thrice with similar results. Oocytes were indicated by arrow heads. Scale bar represents $60 \mu \mathrm{m}$. (b) Histological sections of FOXO3 siRNA-treated porcine ovarian tissues before and after xenografting. Ovarian tissues containing primordial follicles were treated with control $(\mathrm{G})$ or FOXO3 siRNAs (H and I) and xenografted to SCID mice. The tissues were fixed in paraformaldehyde, embedded in methacrylate resin, serially sectioned by $5 \mu \mathrm{m}$, and stained with hematoxylin and eosin. At 2 months after xenografting, follicles developed to the secondary and antral stage in siRNA No. 1- and siRNA No. 3-treated tissues, accompaniment with the oocyte growth ( $\mathrm{K}$ and $\mathrm{L})$. In the similar conditions, primordial follicles did not develop in control siRNA-treated tissues after xenografting $(\mathrm{J})$. ' $\mathrm{r}$ ' indicates mouse renal tissues ( $\mathrm{J}$ and $\mathrm{K})$. Scale bars represent $40(\mathrm{G}-\mathrm{I})$, and $200 \mu \mathrm{m}(\mathrm{J}-\mathrm{L})$.

FOXO3 siRNAs. The degeneration was increased dose dependently by the transfection reagent even in the absence of siRNA (data not shown), indicating that the degeneration was at least partly induced by the toxicity of the transfection reagent. Alternatively, it is possible that $\mathrm{FOXO} 3$ is not the sole factor responsible for the dormancy of primordial oocytes in the pig, and some other treatment might be required for the full activation of those oocytes.

The mechanisms associated with the regulation of FOXO factors have been characterized mainly in somatic cells in response to growth factor signaling via the phosphatidylinositol 3-kinase-protein kinase B (PKB) signaling pathway (Tran et al. 2003). PKB-induced phosphorylation inhibits transcriptional activity of the FOXO members (Biggs et al. 1999, Brunet et al. 1999, Kops et al. 1999, Rena et al. 1999). In the absence of PKB activity, the FOXO members are predominantly localized in the nucleus and are presumed to be active, and PKBmediated phosphorylation induces their relocalization from the nucleus to the cytoplasm (Biggs et al. 1999, Brunet et al. 1999, Brownawell et al. 2001). Functions of FOXO factors are regulated at multiple levels, which include phosphorylation, ubiquitylation, and acetylation (Huang \& Tindall 2007). However, phosphorylation-dependent exclusion of FOXO3 from the nucleus has not been known to be involved in oocytes. How the FOXO3 level increases in prepubertal porcine ovaries is not well understood. Prepubertal porcine ovaries contain a large number of developing follicles (Black \& Erickson 1965). Developing follicles are found in well-vascularized cortical-medullary borders, whereas primordial follicles are located in a relatively avascular region in the cortex (Gougeon 2004). During ovarian development, primordial follicles are placed at the periphery of the ovary, and they might suffer from a shortage of nutrients and growth factors due to poor blood supply. In a worse nutritional condition, the proliferation of cells is arrested in $G_{1}$ or

Table 2 FOXO3 knockdown in porcine primordial oocytes.

\begin{tabular}{lccr}
\hline Treatment & $\begin{array}{c}\text { Number of } \\
\text { tissues } \\
\text { examined }^{\text {a }}\end{array}$ & $\begin{array}{c}\text { Number of } \\
\text { oocytes }\end{array}$ & $\begin{array}{c}\text { Number }(\%) \\
\text { of FOXO3- } \\
\text { positive oocytes }\end{array}$ \\
\hline Control siRNA & 4 & $10 \pm 2^{*}$ & $10 \pm 2\left(96 \pm 2^{*}\right)$ \\
siRNA \# 1 & 4 & $14 \pm 4^{*}$ & $4 \pm 1\left(27 \pm 3^{+}\right)$ \\
siRNA \# 3 & 5 & $19 \pm 3^{*}$ & $7 \pm 1\left(38 \pm 4^{+}\right)$ \\
\hline
\end{tabular}

${ }^{*,+}$ Values with different superscripts are significantly different $(P<0.05)$. ${ }^{a}$ Ovarian tissues measuring $\sim 1 \times 1 \times 0.5 \mathrm{~mm}$ were collected from prepubertal pigs. After FOXO3 siRNA treatment, tissues were cultured for $36 \mathrm{~h}$. Serial cryosections were prepared, reacted with anti-FOXO3 antibody and Alexa Fluor 488-labeled secondary antibody. Sections were counterstained with $\mathrm{PI}$, and FOXO3-positive oocytes were counted throughout the tissues. Each value represents the mean \pm s.E.M. 


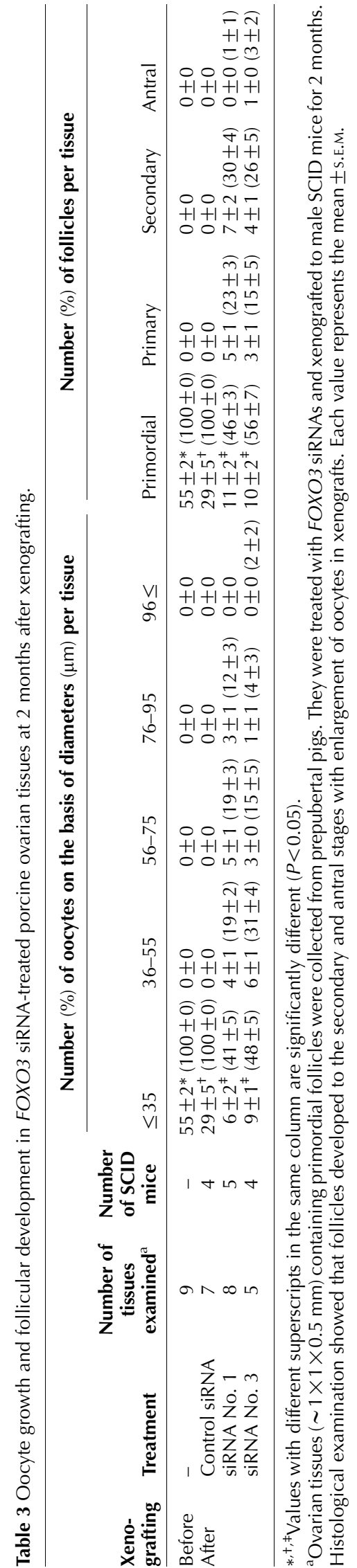

enters a quiescent state $\left(\mathrm{G}_{0}\right)$, and after exposure to nutrients for a prolonged period, the cells return to the $\mathrm{G}_{1}$ stage (Zetterberg \& Larsson 1985). We suppose that primordial oocytes in adult/prepubertal pigs enter such a quiescent state, and they thereby require a long period of stimulation prior to entering the growth phase. $\mathrm{FOXO} 3$ is activated and inhibits cellular growth under the condition of nutrient deprivation in Drosophila (Neufeld 2003). In mammalian cells, nutrient withdrawal activates FOXO3 (Nemoto et al. 2004). In the present experiment, ovarian tissues were xenografted under the kidney capsule where they were exposed to circulatory blood of host mice. The blood provides the factors essential for the metabolic activities of oocytes as well as of other cells in the xenografted tissues. The blood/serum contents (especially growth factors) of host mice may have downregulated FOXO3 in oocytes in the xenografts. Since prepubertal pig oocytes contained a higher level of FOXO3, they are thought to require a longer period to reduce the $\mathrm{FOXO} 3$ in xenografts, and FOXO3 siRNA facilitates this reduction.

In conclusion, our results suggest that primordial oocytes are dormant in the prepubertal pigs by FOXO3related mechanism that functions to establish the nongrowing oocyte pool in the ovary, and that a transient knockdown of FOXO3 activates the primordial oocytes to enter the growth phase.

\section{Materials and Methods \\ Collection of ovarian tissues containing primordial follicles}

Ovaries were collected from 10- to 20-day-old and 6-month-old crossbred (Landrace, Large White and Duroc) gilts. Since the gilts reach the puberty at 6-7 months, we collected ovaries just before puberty and these did not contain corpus luteum. The ovaries were washed in Dulbecco's PBS supplemented with $0.1 \%(\mathrm{w} / \mathrm{v})$ polyvinyl alcohol (PBS-PVA) thrice, and cortical tissues with a thickness of $<0.5 \mathrm{~mm}$ (approximately) were dissected with surgical blades (No. 11; Feather Safety Razor, Osaka, Japan). The tissues were examined under dissection and inverted microscopes. Primordial follicles were identified by diameters within a range of $30-38 \mu \mathrm{m}$ and by oocytes that included a large spherical nucleus surrounded by small lipid droplets (Fig. 1A). Ovarian tissues $(\sim 2 \times 1 \mathrm{~mm})$ that contained primordial follicles were selected and cut into two pieces: one part was fixed immediately for histological examination to assess the number and distribution of different types of follicles, and the other part was immersed in TCM-199 (pH 7.4, Nissui Pharmaceutical Co. Ltd, Tokyo, Japan) containing $0.1 \%(\mathrm{w} / \mathrm{v})$ PVA, $0.85 \mathrm{mg} / \mathrm{ml}$ sodium bicarbonate, and $0.08 \mathrm{mg} / \mathrm{ml}$ kanamycin sulfate (Sigma) and 25 mM HEPES before xenografting.

\section{Xenografting}

Six- to 8-week-old male and female SCID mice were purchased from Clea Japan, Inc., Tokyo, Japan. Prior to xenografting, the mice were anesthetized, and the left kidney was exteriorized 
through a dorsal-horizontal incision. A small hole was torn in the kidney capsule using fine forceps. Three to five pieces of porcine ovarian tissues were inserted underneath the capsule. Half of the female SCID mice were ovariectomized during xenografting. The surgery was performed at room temperature, and the mice were kept on a warming plate $\left(37^{\circ} \mathrm{C}\right)$ for $24 \mathrm{~h}$. The mice were housed in filter-topped cages in a positive pressure room, with free access to clean water and balanced feed pellets. The light cycle of the room was set at $12 \mathrm{~h}$ light:12 h darkness.

Ovarian tissues from both infant and prepubertal pigs were xenografted into male, female, and ovariectomized SCID mice for 2 months at the first phase. However, the xenografting was prolonged up to 6 months in the case of prepubertal pigs. This study was approved by the Institutional Animal Care and Use Committee (Permission number: 15-4-05, 19-5-09 and 19-545) and was carried out according to the Guidelines of Animal Experimentation of Kobe University, Japan.

\section{Histological examination}

Ovarian tissues before and after xenografting were fixed in 3\% $(\mathrm{w} / \mathrm{v})$ paraformaldehyde in PBS, then dehydrated, embedded in methacrylate resin (JB-4; Polysciences, Inc., Warrington, PA, USA), serially sectioned by $5 \mu \mathrm{m}$, and stained with hematoxylin and eosin. The number of different stages of follicles and diameters of the oocytes were recorded. The follicles were counted in every section where the oocyte nucleus was seen. Double counting in adjacent sections was avoided. The follicles were classified into four categories according to the number and morphology of granulosa cell layers: primordial follicles with one layer of flattened granulosa cells surrounding the oocyte, primary follicles with a single layer of cuboidal granulosa cells, secondary follicles with two or more layers of granulosa cells but no antrum, and antral follicles having an antral cavity with multiple layers of granulosa cells. The ovaries from infant pigs contained primordial follicles in that the oocytes were surrounded by the granulosa cells, and in addition, they contained naked oocytes with a few granulosa cells. The latter ones were also classified as primordial follicles. The diameters of the oocytes (excluding the zona pellucida) were measured by taking the average of two perpendicular measurements of each oocyte, which showed the maximum diameter in serial sections, to the nearest $1 \mu \mathrm{m}$ with an ocular micrometer (Nikon, Tokyo, Japan) attached to a microscope.

\section{Collection of primordial oocytes}

Ovarian cortical slices of about $1 \mathrm{~mm}$ thickness were minced into 1-mm square pieces. After washing thrice in $25 \mathrm{mM}$ HEPES-buffered TCM-199 containing $0.1 \%$ (w/v) PVA, $0.85 \mathrm{mg} / \mathrm{ml}$ sodium bicarbonate, and $0.08 \mathrm{mg} / \mathrm{ml}$ kanamycin sulfate, the tissues were incubated for $2.5 \mathrm{~h}$ with gentle agitation in TCM-199 containing $2.5 \mathrm{mg} / \mathrm{ml}$ pronase (actinase E, Kaken Pharmaceutical Co., Tokyo, Japan) at $38.5{ }^{\circ} \mathrm{C}$ under an atmosphere of $5 \% \mathrm{CO}_{2}$ in humidified air. After washing thrice in HEPES-buffered TCM-199, the tissues were gently pipetted for 15-20 min in HEPES-buffered TCM-199 containing $10 \%$ (v/v) FCS (Dainippon Pharmaceutical Co. Ltd,
Osaka, Japan). Oocytes with diameters ranging from 30 to $35 \mu \mathrm{m}$ were picked up using a glass pipette (Fig. 1B). After washing thrice in PBS-PVA, each group of 500 oocytes was transferred into an Eppendorf tube with 2-3 $\mu$ l of PBS-PVA. Then, equal volume of two-times-concentrated SDS sample buffer (Laemmli 1970) was added, and the samples were boiled for $5 \mathrm{~min}$ and kept at $-20{ }^{\circ} \mathrm{C}$ before use.

\section{Preparation of ovarian extracts}

Ovarian cortical tissues containing primordial follicles were collected from prepubertal pigs, and were homogenized by a glass homogenizer by keeping on ice in a lysis buffer containing $50 \mathrm{mM}$ Tris- $\mathrm{HCl}(\mathrm{pH} 7.4), 150 \mathrm{mM} \mathrm{NaCl}, 1 \mathrm{mM}$ EDTA, $0.1 \%$ SDS, $0.5 \mathrm{mM}$-aminophenyl methanesulfonyl fluoride hydrochloride ( $p$-APMSF; Wako Pure Chemical Industries Ltd, Osaka, Japan), and 1\% Triton X-100. Lysates were transferred to an Eppendorf tube to be sonicated by Vibra cell (Sonics \& Materials, Inc., Danbury, CT, USA) with three short bursts of $10 \mathrm{~s}$ at $20 \%$ amplitudes followed by intervals of $1 \mathrm{~min}$ on ice. After centrifugation at $11000 \mathrm{~g}$ for $10 \mathrm{~min}$ at $4{ }^{\circ} \mathrm{C}$, supernatants were collected and protein concentrations were measured using the bicinchoninic acid protein assay. Samples were prepared for western blotting as described above.

\section{Western blotting}

The samples were run on $10 \%$ SDS-PAGE, and the proteins were transferred to hydrophobic PVDF membranes (Immobilon; Millipore Co., Bedford, MA, USA). The membranes were cut into two pieces: one part contained $>50 \mathrm{kDa}$ and the other part contained 50-25 kDa. The membranes were blocked with $10 \%(\mathrm{v} / \mathrm{v})$ FCS in PBS containing $0.1 \%(\mathrm{v} / \mathrm{v})$ Tween 20 (PBS-Tween) for $1 \mathrm{~h}$, and were incubated overnight at $4{ }^{\circ} \mathrm{C}$ in a humidified chamber with rabbit polyclonal anti-human FKHRL1/FOXO3 antibody (1:1000; \# 27567, Upstate Biotechnology, New York, NY, USA; or \# 9467, Cell Signaling Technology, Danvers, MA, USA). It is noted that the FOXO3 is highly conserved between humans and pigs (Fig. 6). After washing thrice in PBS-Tween, the membranes were treated with HRP-conjugated goat anti-rabbit immunoglobulin antibody (1:1000; Pierce Biotechnology, Inc., Rockford, IL, USA) for $1 \mathrm{~h}$ at room temperature. For control, the membranes containing 50-25 kDa were probed with mouse monoclonal anti- $\beta$-actin antibody (1:50 000; \# A2228, Sigma) and subsequently with HRP-conjugated goat anti-mouse immunoglobulin antibody (1:1000; Pierce Biotechnology, Inc). In some experiments, membranes containing $>50 \mathrm{kDa}$ were re-blotted to examine the expression of $\mathrm{MVH}$, a germ cell-specific marker, as an internal control. Membranes were treated with stripping buffer (Re-Blot Plus Strong Solution, Milipore, CA, USA) according to the manufacturer's instructions and reprobed with rabbit polyclonal DDX4/MVH antibody (1:1000; \#13840, Abcam, Cambridge, MA, USA). The granulosa cells were collected from antral follicles and blotted as the control. After washing in PBS-Tween, the peroxidase activity was visualized using the Super Signal detection system (Pierce Biotechnology, Inc). 


\section{Immunohistochemistry}

For immunohistochemistry, cryostat sections of $6 \mu \mathrm{m}$ of cortical tissues from infant and prepubertal ovaries, xenografts (after 4 months), and siRNA-treated ovarian tissues were prepared on silane-coated slides. Sections were dried in air and fixed in 3\% $(\mathrm{w} / \mathrm{v})$ paraformaldehyde in PBS at room temperature for $15 \mathrm{~min}$. To prevent nonspecific antibody binding, blocking was done by $3 \%(\mathrm{w} / \mathrm{v})$ BSA (Wako Pure Chemical Industries Ltd) for $1 \mathrm{~h}$, and immunostaining was performed with rabbit anti-human FKHRL1/FOXO3 antibody (1:250; \#27567, Upstate Biotechnology or $\# 2178$, Sigma) overnight at $4{ }^{\circ} \mathrm{C}$. After washing with PBS, the sections were reacted with Alexa Fluor 488-labeled goat anti-rabbit immunoglobulin antibody (1:1000; Molecular Probes Inc., Eugene, OR, USA) for $45 \mathrm{~min}$, and counterstained with propidium iodide (PI:100 $\mu \mathrm{g} / \mathrm{ml}$; Sigma) for $15 \mathrm{~min}$. Again, after washing thrice with PBS, the sections were mounted with Vectashield Mounting Medium (Vector Laboratories, Inc., Burlingame, CA, USA) and observed under a fluorescence microscope (U-LH100HGAPO; Olympus Optical Co., Tokyo, Japan). Some sections were incubated without a primary antibody as negative controls.

\section{Cloning of partial sequence of FOXO3 and designing FOXO3 SIRNA}

Total RNAs were extracted from porcine fetal testes using ISOGEN RNA extraction reagent (Nippon Gene Inc., Tokyo, Japan), and then total RNAs were reverse-transcribed to cDNA. The cDNAs were amplified by PCR to obtain the fragment of porcine FOXO3. Primers were designed on the basis of human $\mathrm{FOXO3}$ sequence and the available similar sequence in pigs (BP146966 and BX673604): forward 5'-TCTGCCGGCTGGAAGAACTC-3' and reverse 5'-CTACGGATAATGGACTCCAT-3'. PCR amplification conditions consisted of an initial denaturation step at $95{ }^{\circ} \mathrm{C}$ for $2 \mathrm{~min}$, followed by 30 cycles of denaturation at $95{ }^{\circ} \mathrm{C}$ for $1 \mathrm{~min}$, annealing at $55^{\circ} \mathrm{C}$ for $1 \mathrm{~min}$, and extension at $72{ }^{\circ} \mathrm{C}$ for $1.5 \mathrm{~min}$; a final extension period at $72{ }^{\circ} \mathrm{C}$ for 6 min completed the amplification. PCR product was sequenced by Takara Bio Inc. (Shiga, Japan). Using the partial sequence as a template, three sequences of siRNA senses and a nontargeted RNA sequence as a control were designed with online RNAi Designer of Invitrogen as follows: No. 1: 5'-UGAGAUUCAUGGUUCCGGCCAUGUC-3'; No. 2: 5'-UUCAGAGACGAGGGUCCAAACACUG-3'; No. 3: 5'-UUCAAGCUCCCGUUGAACAUGUCCA-3' and control: 5'-GACCCGGAAGGUACCUAAGCUAUCA-3'.

\section{siRNA treatment}

At first, the efficiency of siRNA-induced knockdown was examined in porcine kidney epithelial cell line LLC-PK1 (Dainippon Sumitomo Pharma Co., Ltd, Osaka, Japan). Cells were cultured in TCM-199 containing 5\% (v/v) FCS, $0.1 \mathrm{mg} / \mathrm{ml}$ sodium pyruvate, and $2 \mathrm{nM}$ glutamine (Sigma) at $38.5{ }^{\circ} \mathrm{C}$ under an atmosphere of $5 \% \mathrm{CO}_{2}$ in humidified air. When the cells reached about $50 \%$ confluency, the medium was replaced by a serum-free medium Opti-MEM (Invitrogen) and incubated for $30 \mathrm{~min}$ before transfection. For transfection, $0.25 \%$
Lipofectamine 2000 (Invitrogen), and $0.1 \mu \mathrm{M}$ of each siRNA were added, and the cells were incubated for $6 \mathrm{~h}$ according to the manufacturer's instruction. After transfection, cells were cultured for $36 \mathrm{~h}$ in normal medium. After washing twice with PBS-PVA, cells were detached from the dish by treating with $0.25 \%$ trypsin-EDTA solution (Sigma). They were washed once in normal medium, and thrice in PBS-PVA by centrifugation at $100 \mathrm{~g}$ for $2 \mathrm{~min}$, and were subjected to prepare the sample for western blotting as described above.

Ovarian tissues were treated with 1\% Lipofectamine 2000 and $0.4 \mu \mathrm{M}$ siRNAs following the methods used for cultured cells as described above. Ovarian tissues $(\sim 2 \times 1 \times 0.5 \mathrm{~mm})$ containing primordial follicles were collected from prepubertal pigs. Each tissue was cut into two pieces: one was fixed for immediate histological examination, and the other was allocated for siRNAs treatment (siRNA No. 1 and No. 3). After siRNA treatment for $6 \mathrm{~h}$, some tissues were xenografted to male SCID mice, and the others were further cultured in TCM199 containing $5 \%(\mathrm{v} / \mathrm{v})$ FCS for $36 \mathrm{~h}$ and prepared to detect the FOXO3 expression by immunohistochemistry.

To know the penetration of siRNAs into the ovarian tissues, Alexa Fluor 546-labeled FOXO3 siRNAs (Invitrogen) were transfected in a similar manner. After transfection for $6 \mathrm{~h}$, cryosections were prepared, counterstained with 4,6-diamidino-2-phenylindole (Molecular Probes Inc.), and observed under a fluorescence microscope.

\section{Statistical analysis}

The average numbers of follicles per tissue and average diameters of the oocytes were represented as the mean \pm s.E.M. All data were subjected to one-way ANOVA, and the significance of difference among means was determined by the Tukey's multiple range test. All percentile data were transformed to arc sin values before analysis. Differences at $P<0.05$ were considered statistically significant.

\section{Declaration of interest}

The authors declare that there is no conflict of interest that could be perceived as prejudicing the impartiality of the research reported.

\section{Funding}

This work was supported in part by the Grant-in-Aid for Scientific Research of the Japan Society for the Promotion of Science to T Miyano and M Moniruzzaman, and by the $21 \mathrm{st}$ Century COE program to J Lee from the Ministry of Education, Culture, Sports, Science, and Technology of Japan.

\section{References}

Abdelrahim M, Smith R III \& Safe S 2003 Aryl hydrocarbon receptor gene silencing with small inhibitory RNA differentially modulates Ah-responsiveness in MCF-7 and HepG2 cancer cells. Molecular Pharmacology 63 1373-1381. 
Alvarez B, Martinez A, Burgering BM \& Carrera AC 2001 Forkhead transcription factors contribute to execution of the mitotic programme in mammals. Nature 413 744-747.

Biggs WH III, Meisenhelder J, Hunter T, Cavenee WK \& Arden KC 1999 Protein kinase B/Akt-mediated phosphorylation promotes nuclear exclusion of the winged helix transcription factor FKHR1. PNAS 96 7421-7426.

Black JL \& Erickson BH 1965 Oogenesis and ovarian development in the prenatal pig. Anatomical Record 161 45-56.

Bosma MJ \& Carroll AM 1991 The SCID mouse mutant: definition, characterization, and potential uses. Annual Review of Immunology $\mathbf{9}$ 323-350.

Bosma GC, Custer RP \& Bosma MJ 1983 A severe combined immunodeficiency mutation in the mouse. Nature 301 527-530.

Brenkman AB \& Burgering BM 2003 FOXO3 eggs on fertility and aging. Trends in Molecular Medicine 9 464-467.

Brownawell AM, Kops GJ, Macara IG \& Burgering BM 2001 Inhibition of nuclear import by protein kinase $B$ (Akt) regulates the subcellular distribution and activity of the forkhead transcription factor AFX. Molecular and Cellular Biology 21 3534-3546.

Brunet A, Bonni A, Zigmond MJ, Lin MZ, Juo P, Hu LS, Anderson MJ, Arden KC, Blenis J \& Greenberg ME 1999 Akt promotes cell survival by phosphorylating and inhibiting a forkhead transcription factor. Cell $\mathbf{9 6}$ 857-868.

Candy CJ, Wood MJ \& Whittingham DG 1995 Follicular development in cryopreserved marmoset ovarian tissue after transplantation. Human Reproduction 10 2334-2338.

Castrillon DH, Miao L, Kollipara R, Horner JW \& DePinho RA 2003 Suppression of ovarian follicle activation in mice by the transcription factor FOXO3. Science 301 215-218.

Eppig JJ \& O'Brien MJ 1996 Development in vitro of mouse oocytes from primordial follicles. Biology of Reproduction 54 197-207.

Fortune JE, Cushman RA \& Kito WS 2000 The primordial to primary follicle transition. Molecular and Cellular Endocrinology 163 53-60.

Gampel A \& Mellor H 2002 Small interfering RNAs as a tool to assign Rho GTPase exchange-factor function in vivo. Biochemical Journal 366 393-398.

Gosden RG, Boulton MI, Grant K \& Webb R 1994 Follicular development from ovarian xenografts in SCID mice. Journal of Reproduction and Fertility 101 619-623.

Gougeon A 2004 Dynamics of human follicular growth: morphologic, dynamic, and functional aspects. In The Ovary, 2 edn, pp 25-43. Eds PCK Leung \& EY Adashi. California: Elsevier Academic Press.

Hirshfield AN 1991 Development of follicles in the mammalian ovary. International Review of Cytology 124 43-101.

Huang H \& Tindall DJ 2007 Dynamic foxo transcription factors. Journal of Cell Science $1202479-2487$.

Kaneko H, Kikuchi K, Noguchi J, Hosoe M \& Akita T 2003 Maturation and fertilization of porcine oocytes from primordial follicles by a combination of xenografting and in vitro culture. Biology of Reproduction 69 1488-1493.

Kops GJ, de Ruiter ND, De Vries-Smits AM, Powell DR, Bos JL \& Burgering BM 1999 Direct control of the forkhead transcription factor AFX by protein kinase B. Nature 398 630-634

Laemmli UK 1970 Cleavage of structural proteins during the assembly of the head of bacteriophage T4. Nature 227 680-685.

Lee J, Kitajima TS, Tanno Y, Yoshida K, Morita T, Miyano T, Miyake M \& Watanabe Y 2008 Unified mode of centromeric protection by shugoshin in mammalian oocytes and somatic cells. Nature Cell Biology 10 42-52.

Lintern-Moore S \& Moore GP 1979 The initiation of follicle and oocyte growth in the mouse ovary. Biology of Reproduction 20 773-778.
Mattiske D, Shaw G \& Shaw JM 2002 Influence of donor age on development of gonadal tissue from pouch young of the tammar wallaby, Macropus eugenii, after cryopreservation and xenografting into mice. Reproduction 123 143-153.

McGee EA \& Hsueh AJ 2000 Initial and cyclic recruitment of ovarian follicles. Endocrine Reviews 21 200-214.

Miyano T 2003 Bringing up small oocytes. Theriogenology 59 61-72.

Moniruzzaman M \& Miyano T 2007 KIT-KIT ligand in the growth of porcine oocytes in primordial follicles. Journal of Reproduction and Development 53 1273-1281.

Nakamura N, Ramaswamy S, Vazquez F, Signoretti S, Loda M \& Sellers WR 2000 Forkhead transcription factors are critical effectors of cell death and cell cycle arrest downstream of PTEN. Molecular and Cellular Biology 20 8969-8982.

Nemoto S, Fergusson MM \& Finkel T 2004 Nutrient availability regulates sirt1 through a forkhead-dependent pathway. Science 306 2105-2108.

Neufeld TP 2003 Shrinkage control: regulation of insulin mediated growth by FOXO transcription factor. Journal of Biology 218.

Oktay K, Newton H, Mullan J \& Gosden RG 1998 Development of human primordial follicles to antral stages in SCID/hpg mice stimulated with follicle stimulating hormone. Human Reproduction 13 1133-1138.

Oxender WD, Colenbrander B, Van dewiel DFM \& Wensing CJG 1979 Ovarian development in fetal and prepubertal pigs. Biology of Reproduction 21 715-721.

Peters H 1969 The development of the mouse ovary from birth to maturity. Acta Endocrinologica 62 98-116.

Reddy P, Shen L, Ren C, Boman K, Lundin E, Ottander U, Lindgren P, Liu YX, Sun QY \& Liu K 2005 Activation of Akt (PKB) and suppression of FKHRL1 in mouse and rat oocytes by stem cell factor during follicular activation and development. Developmental Biology 281 160-170.

Rena G, Guo S, Cichy SC, Unterman TG \& Cohen P 1999 Phosphorylation of the transcription factor forkhead family member FKHR by protein kinase B. Journal of Biological Chemistry 274 17179-17183.

Russe I 1983 Oogenesis in cattle and sheep. Bibliotheca Anatomica 24 77-92.

Senbon S, Ota A, Tachibana M \& Miyano T 2003 Bovine oocytes in secondary follicles grow and acquire meiotic competence in severe combined immunodeficient mice. Zygote 11 139-149.

Tran H, Brunet A, Griffith EC \& Greenberg ME 2003 The many forks in FOXO's road. Science's STKE: Signal Transduction Knowledge Environment 172 RE5.

Wang C \& Roy SK 2006 Expression of growth differentiation factor 9 in the oocytes is essential for the development of primordial follicles in the hamster ovary. Endocrinology 147 1725-1734.

Weigel D \& Jackle H 1990 The fork head domain: a novel DNA binding motif of eukaryotic transcription factors? Cell 63 455-456.

Yamaguchi YL, Tanaka SS, Yasuda K, Matsui Y \& Tam PP 2006 Stagespecific importin13 activity influences meiosis of germ cells in the mouse. Developmental Biology 297 350-360.

Zetterberg A \& Larsson O 1985 Kinetic analysis of regulatory events in G1 leading to proliferation or quiescence of swiss 3T3 cells. PNAS 82 5365-5369.

Received 21 May 2009

First decision 6 July 2009

Revised manuscript received 15 October 2009

Accepted 30 October 2009 